\title{
P-232L: Late-News Poster: New Results on Bistable Photoaligned Ferroelectric LCDs: Grey Scale Generation, Stabilization and Passive Driving Scheme Xihua Li, Anatoli Muravsky, Alexander Muravsky, Hon Lung Cheung, Peizhi Xu, Eugene Pozhidaev and Vladimir Chigrinov
}

\author{
Center for Display Research, Department of Electrical and Electronic Engineering \\ Hong Kong University of Science and Technology \\ Clear Water Bay, Kowloon, Hong Kong
}

\begin{abstract}
The principles for intrinsic greyscale generation and stabilization of bistable photoaligned FLCD are discussed and investigated. In order to realize these principles, the passive matrix driving scheme for FLCD is proposed and studied. Passive $160 \times 160$ matrix addressing photoaligned $5 \mu m$ reflective FLC display $(48 \mathrm{~mm} \times 46 \mathrm{~mm})$ with high contrast and 4 memorized grey scale levels is demonstrated. Images can be saved for very long time without any power supply. $15 \mathrm{~V}$ driving voltage is only needed to refresh information, which is impressive and desirable for low power consumption display such as PDA, e-paper etc.
\end{abstract}

\section{Introduction}

Bistable ferroelectric liquid crystal displays (FLCD) are well known for a long time, however, the generation of the memorized grey scale was always an issue ${ }^{[1]}$. Common surface stabilized FLC structure with a bistable switching can have a memorized black and white states, but cannot provide an intrinsic grey scale, unless a time or space averaging process is applied ${ }^{[2]}$.

FLC cells were aligned with ordinary rubbing technique for many years ${ }^{[3]}$. However, "zig-zag defects" appear making difficult to get the good memory effects in the case of low pretilt angle in FLC cells prepared by rubbing. Our group has already demonstrated the high uniformity of the FLC layer obtained by photoalignment technology ${ }^{[4]}$. The photoaligned material we used is the azobenzene sulfuric dye SD-1, which chemical structure is shown in Fig. 1.

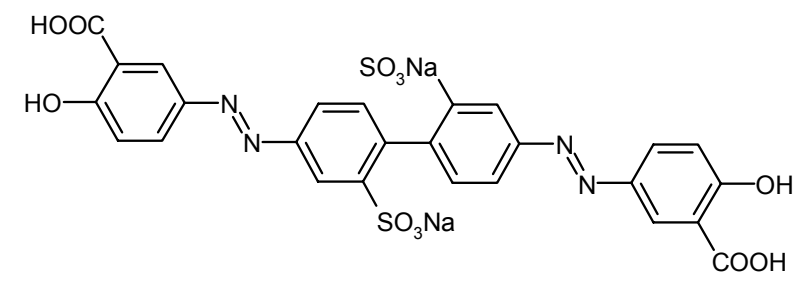

Fig 1. Structure of azo-dye SD-1

A remarkable property of this azo-dye is the pure reorientation of the molecular absorption oscillators perpendicular to the UV light polarization, which is not accompanied with photochemical transformations. Thus a good chance to increase the chemical stability of photo aligning SD-1 layers and to prevent the degradation of bistable FLC switching is provided ${ }^{[4,5]}$. In our experiments, the photoaligned FLC cell shows CR $>500: 1$ at the wavelength $\lambda=0.63 \mu \mathrm{m}$ both in surface stabilized FLC (SSFLC) and helix FLC electrooptical modes using binary switching pulse $^{[4]}$.

CITIZEN WATCH Co. has already demonstrated low power consumption displays using FLC with memory effects ${ }^{[6]}$. They use $\mathrm{SiO}$ or $\mathrm{SiO}_{2}$ films for alignment layer and photolithographic 1.5um spacers for fabrication, which gives much difficulty for fabrication process and increases the cost of displays. Compared with SIO alignment film, photoaligned film can provide more uniform, better and "cleaner" alignment quality for FLC display. Large cell gap can be used in our FLC display, which is more interesting for display companies. Furthermore, there is no report about grey scale generation from their group.

In this paper, we would like to report our latest progress on FLC display using photoalignment technology. New FLC material (FLC-514, $\left.\quad \mathrm{P}_{\mathrm{s}}>90 \mathrm{nc} / \mathrm{cm}^{2}, \quad \mathrm{SmC}^{*} \rightarrow \rightarrow^{71^{\circ} \mathrm{C}} \rightarrow \mathrm{SmA} \rightarrow{ }^{85^{\circ} \mathrm{C}} \rightarrow \mathrm{Is}\right) \quad$ is employed in experiments, which is developed by Dr. E. Pozhidaev from P.N.Lebedev Physical Institute in Moscow. We will investigate the methods how to generate and stabilize grey scale levels using passive multiplex driving in FLCD. Based on this, $160 \times 160$ passive driving addressed 5 um reflective FLCD with 4 memorized greyscale levels is demonstrated using photoaligned technology.

\section{Experiments}

Figure 2 shows the cross section of our FLC display.

\begin{tabular}{|c|}
\hline Substrate \\
\hline ITO \\
\hline $0.4 \%$ SD-1 \\
\hline FLC \\
\hline $0.4 \%$ SD- 1 \\
\hline ITO \\
\hline Substrate \\
\hline
\end{tabular}

Fig 2. Cross section of photoaligned FLC display using SD-1 
The two glass substrates with Indium Tin Oxide (ITO) are covered with $0.4 \%$ SD-1 in DMF. After spin coating, both substrates are baked on hot plate to remove DMF solvent. After this, the two substrates were illuminated under polarized UV light (500 Wt Hg lamp with interferometric filter, $\lambda_{\exp }=365 \mathrm{~nm}, \mathrm{P}_{\exp }$ $=2.3 \mathrm{mWt} / \mathrm{cm}^{2}$ ) to get parallel alignment. After assembled with spacers, FLC-514 is injected into the empty cell. Finally, the cell was slowly cooled down to room temperature and FLC phase transformed from SmA to $\mathrm{SmC}^{*}$.

\section{Results and Discussions 3.1 Principle of grey scale generation for \\ FLC}

The memorized grey scale of passively addressed FLCD can be obtained, if FLC posses a high spontaneous polarization $\mathrm{P}_{\mathrm{s}}>50$ $\mathrm{nC} / \mathrm{cm}^{2}$, when ferroelectric domains exist, being one of possible reasons for the grey scale ${ }^{[7]}$.Fig. 3 shows the FLC texture of the memorized grey scale levels observed under crossed polarizers after switching off the applied voltage. The grey scale appears as a result of the spatial averaging of "black" and "white" areas in light trasnsmission.

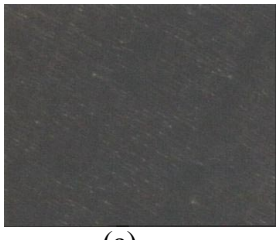

(a)

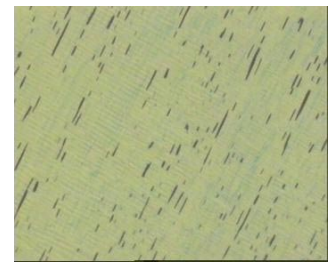

Fig 3. Textures of $5 \mu \mathrm{m}$ FLC memorized grey scale level after switching off the pulse with amplitudes of (a) $0 \mathrm{~V}$, (b) $3 \mathrm{~V}$, (c) $4 \mathrm{~V}$, (e) $6 \mathrm{~V}$ and (e) $15 \mathrm{~V}$. FLC dark state was supposed to be unselected state.

Owing to hysteresis loop for FLC, a special signal should be designed to generate FLC gray scale (Fig. 4).

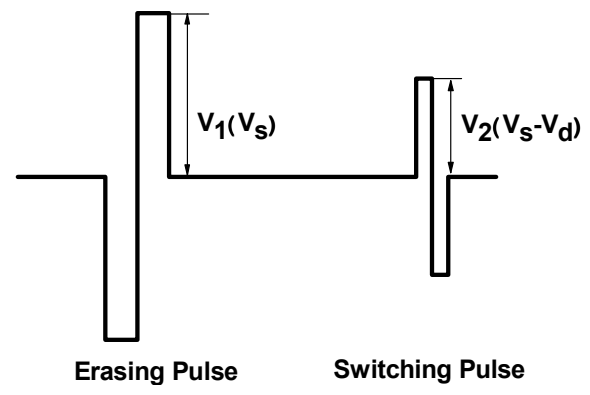

Fig 4. Driving waveform for TVC measurement of FLC.
First pulse is erasing one, which has large enough amplitude and a duration time to change the state to "dark" (or "bright"). Second pulse is selecting one, which amplitude is changing for grey scale generation. Fig. 5 shows the TVC response of 5um Photoaligned FLC cell for normal black modes, which means erasing to black state for the first pulse. The amplitude of the switching pulse varies from $11 \mathrm{v}$ to $16 \mathrm{~V}$, while the duration time is $1 \mathrm{~ms}$. The result shows that it is possible to generate many gray scale levels without crosstalk.

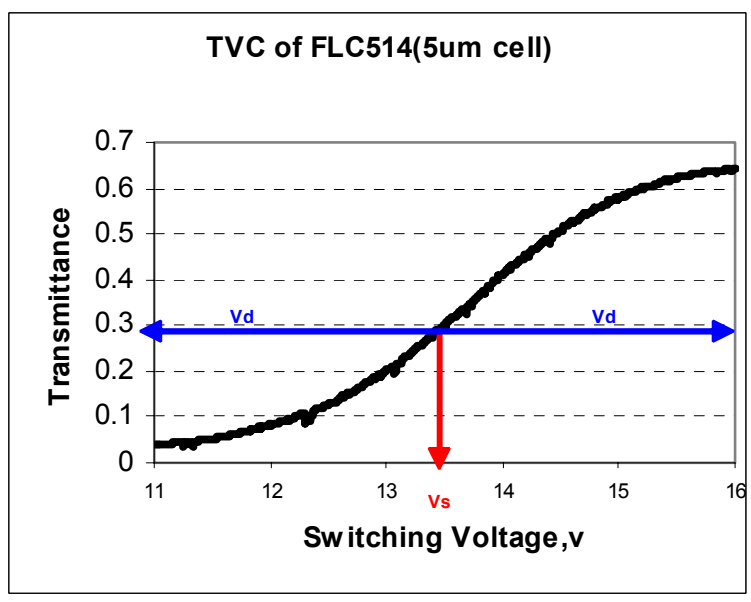

Fig 5. TVC of $5 \mu \mathrm{m}$ FLC 514 cell (duration time is $1 \mathrm{~ms}$ for switching pulse)

\subsection{Grey scale stabilization under passive multiplex driving}

Gray scale levels in passive matrix driving FLC display, can be stabilized even with a crosstalk. From TVC response of $5 \mu \mathrm{m}$ FLC cell without crosstalk (Fig.5), 13.5V and $1 \mathrm{~ms}$ switching pulse can let the FLC select to a $50 \%$ of a gray scale level. We used $11 \mathrm{~V}$ pulse for nonselected state and $16 \mathrm{~V}$ is for the selected state, and $2.5 \mathrm{~V}$ and $1 \mathrm{~ms}$ pulse is employed as column signal (data) to address different levels of a gray scale. So we may get many as possible stabilized gray scale levels, having the maximum contrast ratio at the same time.

Fig. 6 shows the TVC response of the same $5 \mu \mathrm{m}$ Photoaligned FLC cell with crosstalk of $2.5 \mathrm{~V}$ amplitude and $1 \mathrm{~ms}$ duration time for a multiplex ratio of 60:1. The amplitude of the switching pulse $\left(\mathrm{V}_{2}\right)$ varies from $11 \mathrm{v}$ to $16 \mathrm{~V}$ with the same duration time (1ms). The maximum transmittance variation for the gray scale level (nonstabilized level) is about 0.1 while the total transmittance between selected and nonselected states is about 0.6. It means at least about 6 stabilized grey scale levels can be received using the multiplex ratio of $60: 1$. The contrast ratio is about $30: 1$ in this case. 
TVC of 5um FLC514 cell with crosstalk 2.5V

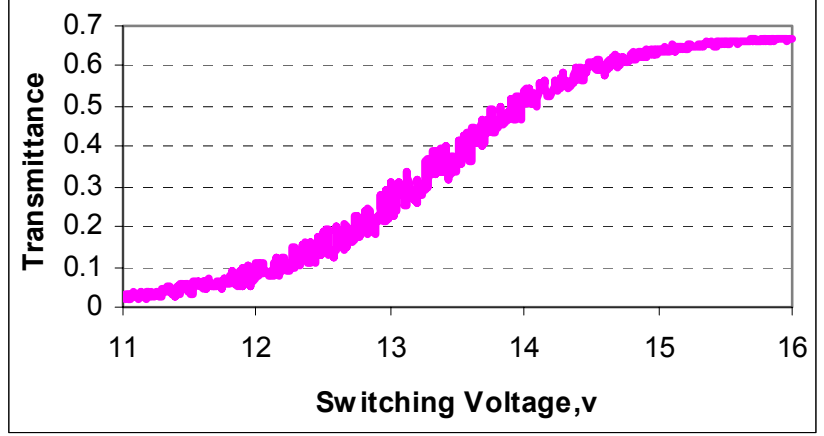

Fig 6. TVC of 5um FLC 514 cell with multiplex ratio 60:1 (duration time is $1 \mathrm{~ms}$ for the switching pulse)

\subsection{Passive driving scheme}

As FLCD can memorize images for very long time, power supply is only needed for refreshing information on display. To write new images, two pulse frames showed in Figure 7 are used. First frame is to reset all pixels to black (or white) state. No column voltage is applied on the display. Second frame is for grey scale generation. Time between these two pulses can be very short, while it was equal to one frame time used in our driving scheme.

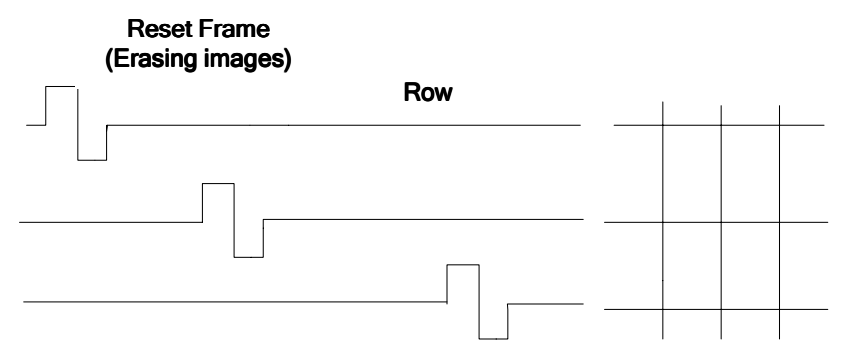

(a) Reset Frame

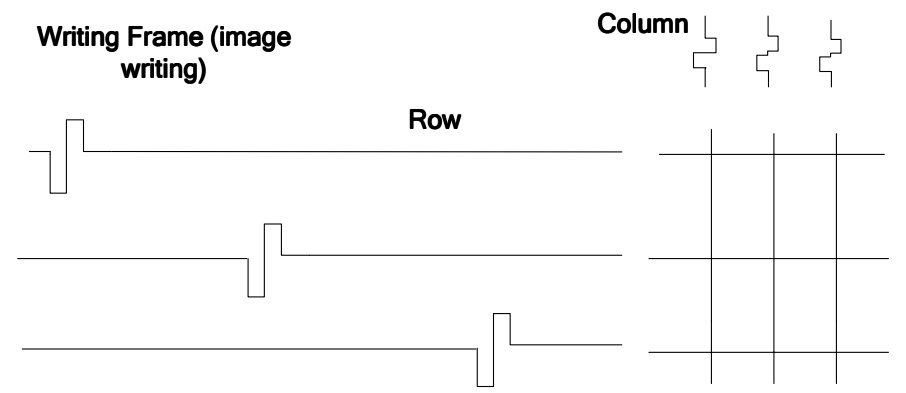

(b) Writing Frame

Fig 7. Two pulse frames of passive multiplex driving scheme for FLC display
As a result, there will be two pulses for row driving (Figure 8). Erasing pulse has same amplitude $\left(\mathrm{V}_{\mathrm{s}}\right)$ but two times long duration time compared with writing pulse. For column driving, the pulse duration is the same $(\mathrm{T})$ and the amplitude is $\mathrm{V}_{\mathrm{d}}$. If $\mathrm{V}_{\mathrm{s}} / \mathrm{V}_{\mathrm{d}}=2$, this driving scheme is equal to SEIKO standard for STN passive multiplex driving ${ }^{[1]}$. In order to generate grey scale, the pulse width modulation (PWM) method for column driving is proposed. The ratio of PWM can be defined as $\lambda / T$, where $\tau$ is the time interval in the column pulse. Variation of $\lambda / \mathrm{T}\left(0 \leq \frac{\lambda}{T} \leq 1\right)$ can provide different grey scale levels.

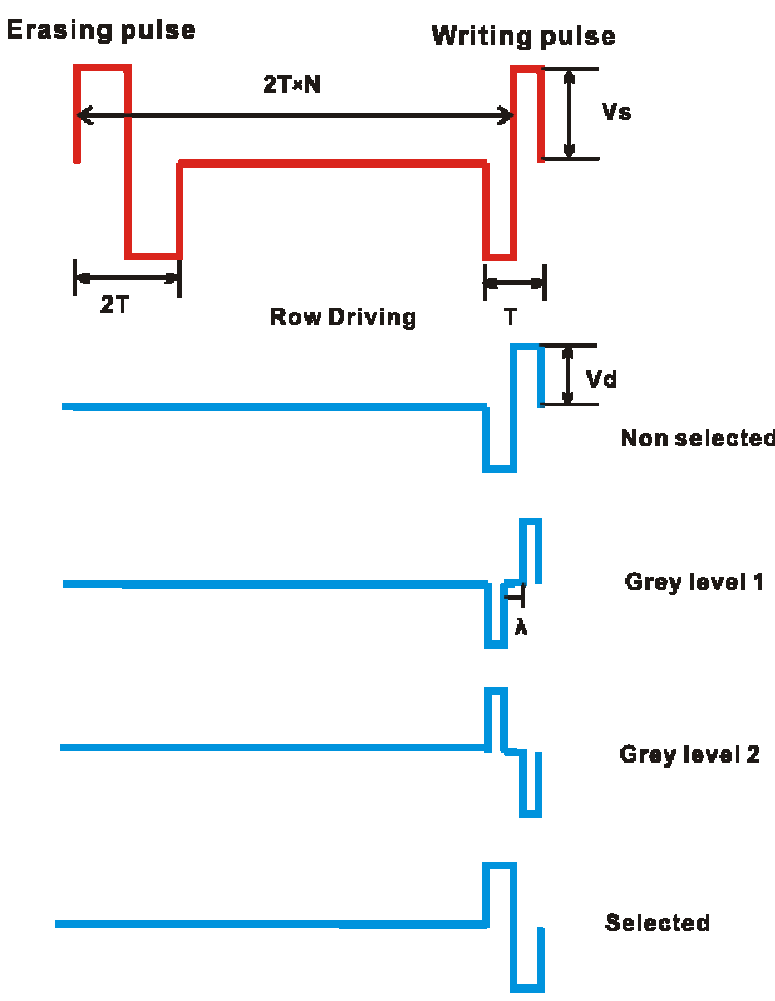

Column Driving

Fig 8. Passive multiplex driving Scheme for bistable FLC display (N: lines of row)

Based on this, $160 \times 160$ passive matrix addressing FLC display ((the size of about $48 \mathrm{~mm} \times 44 \mathrm{~mm}$ ) is demonstrated. Fig. 9 shows the texture of nonselected (bright) and selected (dark) regions, which shows very uniform alignment quality and high contrast ratio. 


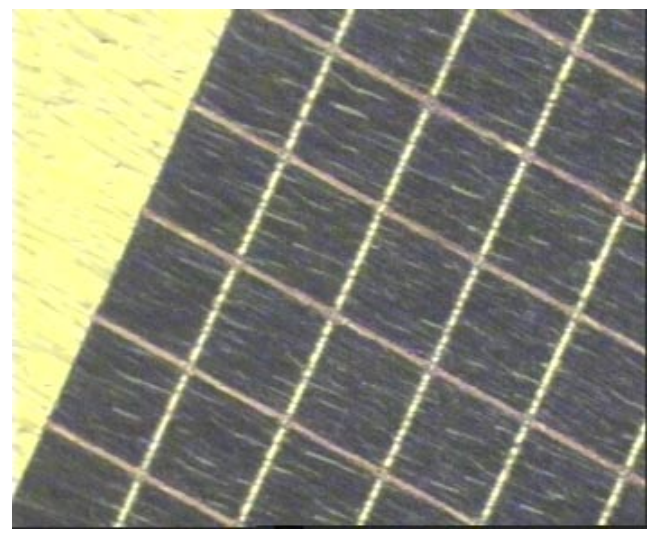

Fig 9. Textures of nonselected (bright) and selected (dark) regions of the FLCD

Comparable with common TN LCD driving, power supply is not needed when operating. Fig.10 shows the original and the same Image on our bistable reflective FLCD with 4 memorized grey scale levels. Owing to a birefringence for $5 \mu \mathrm{m}$ cell gap, the selected and nonselected states are green and dark for our FLC display, which is green background in the image. The images can be saved for infinitive time without any power supply.

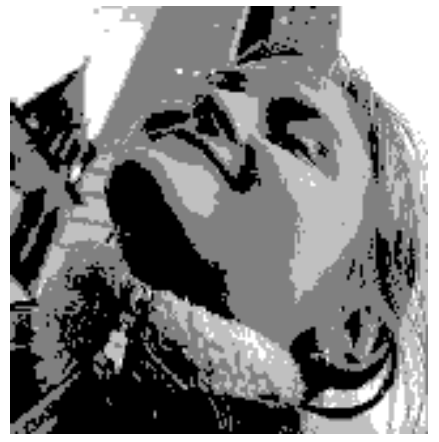

(a)Original picture

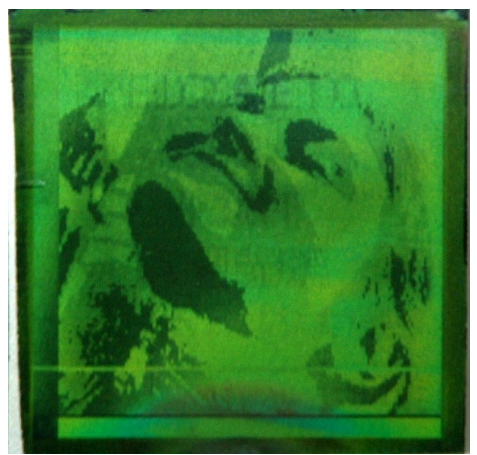

(b) Image showed on display without any backlight

Fig 10. Original picture and the same Image showed on the $160 \times 160$ passive matrix addressing $5 \mu \mathrm{m}$ reflective FLCD using photoaligned technology with four memorized grey scale levels

\section{Conclusions}

In this paper, principles and methods for grey scale generation and stabilization of photoaligned bistable FLC display are investigated and discussed. Based on this, the passive driving scheme is proposed for stabilized greyscale levels. $160 \times 160$ passive matrix addressed bistable $5 \mu \mathrm{m}$ reflective FLC display is demonstrated with the driving voltage of $13 \mathrm{~V}$ and 5 um cell gap. Images can be saved for very long time without any power supply.

We discuss the principles for intrinsic gray scale generation and gray levels stabilization using passive matrix driving methods for FLC display. We believed, our result is a breakthrough for bistable FLC display with a low power consumption, which is the best solution for watches, PDA or e-papers in the future.

\section{Acknowledgements}

This research was supported by ITF grant ITS/111/03 from Hong Kong government.

\section{References}

[1] V.G.Chigrinov: Liquid Crystal Devices: Physics and Applications (Artech House, Boston, London, 1999).

[2] S.T.Lagerwall: Ferroelectrics 301 (2004) 15.

[3] Y. Inaba, K.Katagiri, H. Inoue, J. Kanbe, S. Yoshihara, S. Iijuma, "Essential factors in high-duty FLC matrix display", Ferroelectrics, 85, 643-652 (1988).

[4] E. Pozhidaev, V. Chigrinov, D. Huang, A. Zhukov, Jacob Ho and H. S. Kwok, "Photoalignment of Ferroelectric Liquid Crystals by Azodye Layers", Japanese Journal of Applied Physics, Vol. 43, No. 8A, pp. 5440-5446, 2004.

[5] H. Takada, H. Akiyama, and H. Takatsu, V. Chigrinov, E. Prudnikova, V. Kozenkov, and H. S. Kwok, "Aligning Layers Using Azo Dye Derivatives for Liquid Crystal Devices”, SID’03 Digest, pp. 620-623, 2003

[6] S. Kondoh, A. Suguro, K. Noguchi, K. Iio, K. Ueda, N. Takahashi, and M. Fujino, " Low Power Consumption Displays using Ferroelectric Liquid Crystals”, IDW'05 Digest, pp.81-82, 2005.

[7] E.P.Pozhidaev, A.L. Andreev and I.N. Kompanets: Proc. of $7^{\text {th }}$ Int. Conf. on FLC, p.164, 1999 